\title{
Combination chemotherapy with TAS-102 plus bevacizumab in salvage-line treatment of metastatic colorectal cancer: A single-center, retrospective study examining the prognostic value of the modified Glasgow Prognostic Score in salvage-line therapy of metastatic colorectal cancer
}

\author{
NOBUHISA MATSUHASHI ${ }^{1}$, TAKAO TAKAHASHI $^{1}$, HIRONORI FUJI $^{2}$, TOMONARI SUETSUGU $^{1}$, \\ MASAHIRO FUKADA $^{1}$, YOSHINORI IWATA ${ }^{1}$, YOSHIHISA TOKUMARU ${ }^{1}$, TAKEHARU IMAI ${ }^{1}$, \\ RYUTARO MORI ${ }^{1}$, TOSHIYUKI TANAHASHI ${ }^{1}$, SATOSHI MATSUI ${ }^{1}$, HISASHI IMAI ${ }^{1}$, \\ YOSHIHIRO TANAKA ${ }^{1}$, KAZUYA YAMAGUCHI ${ }^{1}$, MANABU FUTAMURA ${ }^{1}$ and KAZUHIRO YOSHIDA ${ }^{1}$ \\ ${ }^{1}$ Department of Surgical Oncology, Gifu University School of Medicine; \\ ${ }^{2}$ Department of Pharmacy, Gifu University Hospital, Gifu 501-1194, Japan
}

Received August 27, 2018; Accepted June 14, 2019

DOI: $10.3892 /$ mco.2019.1899

\begin{abstract}
The combination regimen of TAS-102, a novel oral nucleoside antitumor agent containing trifluridine and tipiracil hydrochloride, with bevacizumab (C-TASK FORCE), a selective monoclonal antibody inhibitor of vascular endothelial growth factor-A, as salvage-line therapy for metastatic colorectal cancer (mCRC) was established based on its high clinical effectiveness. The aim of the present study was to evaluate the prognostic accuracy of the modified Glasgow Prognostic Score (mGPS) in patients receiving TAS-102 plus bevacizumab. The study included 17 patients (12 men and 5 women, mean age $60.4 \pm 13.4$ years) with unresectable $\mathrm{mCRC}$ who were confirmed to have wild-type or mutant RAS genes. The patients received salvage-line treatment with TAS-102 plus bevacizumab at the Surgical Oncology Department of Gifu University School of Medicine between March 2016 and August 2018. The study population was heavily pretreated; the majority of the patients $(71 \%)$ had received $\geq 4$ prior regimens and, in addition to fluoropyrimidine, irinotecan and oxaliplatin, all had received bevacizumab (100\%) and either cetuximab or panitumumab (47\%). The RAS status was wild-type in $9(53 \%)$ and mutant in $8(47 \%)$ patients. The primary tumor locations included the right-sided colon in 5 patients (29\%; cecum in 2 and transverse colon in 3 cases) and left-sided colorectum in 12 patients [71\%; sigmoid colon in 4, rectosigmoid (Rs) in 4, and
\end{abstract}

Correspondence to: Professor Kazuhiro Yoshida, Department of Surgical Oncology, Gifu University School of Medicine, Yanagido 1-1, Gifu 501-1194, Japan

E-mail:kyoshida@gifu-u.ac.jp

Key words: TAS-102 plus bevacizumab, metastatic colorectal cancer, salvage-line chemotherapy, Glasgow Prognostic Score rectum above/below the peritoneal reflection $(\mathrm{Ra} / \mathrm{b})$ in 4 cases]. Metastatic sites included the liver in 15 (88\%), lung in 13 (76\%), lymph nodes in 7 (41\%), and peritoneal dissemination in 5 (24\%) patients. The number of metastatic sites was 1 in $3(18 \%)$ and $>2$ in $14(82 \%)$ patients. Their first staging imaging scans (after 2 cycles of therapy) were available for review in all 17 patients. At first evaluation, 5 (29\%) patients had progressive disease (PD), $12(71 \%)$ had stable disease, and none had a partial response to TAS-102 plus bevacizumab. The median overall survival (OS) of 14.1 months and progression-free survival (PFS) of 6.8 months were comparable to the 11.2 and 5.6 months, respectively, in the C-TASK FORCE study. Upon considering three groups, namely mGPS 0 , mGPS 1 and mGPS 2, the median PFS times were significantly different (mGPS 0 vs. mGPS $2, P=0.02$; and mGPS 1 vs. mGPS $2, \mathrm{P}=0.06$ ). The median PFS times in the mGPS 0,1 and 2 groups were 12.1, 4.8 and 2.3 months, respectively. Median OS was also significantly different (mGPS 0 vs. mGPS 2, $\mathrm{P}=0.01$; and mGPS 1 vs. mGPS 2, P=0.04). The median OS times in the mGPS 0,1 and 2 groups were 14.0, not reached, and 2 months, respectively. The present study demonstrated the efficacy and safety of the TAS-102 plus bevacizumab combination as salvage-line treatment. This combination therapy (the TAS-102 plus bevacizumab) has obtained valid results with PFS OS as well as C-TASK.FORCE study. The results of the present study also confirmed the prognostic accuracy of mGPS in salvage-line treatment of patients with mCRC.

\section{Introduction}

The incidence and mortality rates of colorectal cancer (CRC) vary markedly worldwide. Globally, CRC is the third most commonly diagnosed cancer in men and the second in women (1), with 1.8 million new cases and almost 861,000 deaths in 2018 according to the World Health Organization GLOBOCAN database $(2,3)$. 
Approximately $25 \%$ of patients with CRC have metastatic disease, with a clinically significant detrimental effect on prognosis $(4,5)$. With the administration of chemotherapy and molecular targeted therapy, the median overall survival (OS) time of metastatic CRC (mCRC) has improved from 12 to 34.9 months $(6,7)$. However, mCRC carries a poor prognosis and cannot be cured with the currently available therapy options. Chemotherapy designed to prolong survival and improve the quality of life of patients is the mainstay of treatment (8).

TAS-102 (trifluridine and tipiracil hydrochloride, a novel combination oral nucleoside antitumor agent) was first approved in Japan in March 2014 and received US Food and Drug Administration (FDA) approval in September 2015, after an international phase III clinical trial in patients with refractory metastatic colon cancer demonstrated an OS benefit with TAS-102 compared with placebo (9).

Bevacizumab is a selective monoclonal antibody inhibitor of vascular endothelial growth factor (VEGF)-A; it was FDA-approved for $\mathrm{mCRC}$ in 2005, after showing efficacy in combination with 5-fluorouracil (5-FU)-based chemotherapy $(10,11)$. The combination regimen of a fluoropyrimidine with bevacizumab in $\mathrm{mCRC}$ in the first-line setting was established on the basis of high clinical effectiveness and no overlapping toxicity between agents. Similarly, combining TAS-102 with bevacizumab may be beneficial. Evidence of the activity of TAS-102 plus bevacizumab in MCRC was reported in a phase I/II trial of the C-TASK FORCE study (12). In addition, in a phase II/III trial of the TRUSTY study was initiated in 2017 and the study is anticipated to be completed in 2022 (13). The aim of the present cohort study was to evaluate the benefits of using the modified Glasgow Prognostic Score (mGPS) as an inflammatory index and the combination of TAS-102 with bevacizumab as salvage-line treatment.

\section{Patients and methods}

Patients. This study included 17 patients with unresectable mCRC who were confirmed to have the wild-type or mutant RAS gene. The patients received salvage-line treatment with TAS-102 plus bevacizumab at the Surgical Oncology Department of Gifu University School of Medicine between March 2016 and August 2018. The level 1 dose was TAS-102 (Taiho Pharmaceutical Co., Ltd.) at $35 \mathrm{mg} / \mathrm{m}^{2}$ body surface area, administered orally twice per day on days $1-5$ and 8-12 of a 28 -day cycle, plus bevacizumab at $5 \mathrm{mg} / \mathrm{kg}$ body weight, administered by intravenous infusion for $30 \mathrm{~min}$ every 2 weeks. Tumor shrinkage in the 17 patients was evaluated according to the Response Evaluation Criteria in Solid Tumors (RECIST) version 1.1, based on the dose-limiting toxicity observed in each cycle. The demographic and disease characteristics of the patients were also recorded. On the basis of the results of previous clinical studies (9), bevacizumab plus standard regimens, such as FOLFOX, CAPOX and FOLFIRI, is currently recommended as first-line treatment for mCRC (Table I).

Written informed consent was obtained from all patients enrolled in the present study. The study protocol conformed to the ethical guidelines of the 1975 Declaration of Helsinki and the guidelines of the regional ethical committees of Zurich and Basel, Switzerland, and was approved by the Institutional Review Board of the Gifu University Graduate School of Medicine (no. 28-196).

RECIST 1.1 guideline. Computed tomography (CT) is currently considered the best and most reproducible method for measuring lesions when assessing response. The RECIST guideline defines measurability of lesions on CT scan based on a slice thickness of $\leq 5 \mathrm{~mm}$. When the CT slice thickness is $>5 \mathrm{~mm}$, the minimum size of a measurable lesion must be at least twice the slice thickness.

Evaluation of target lesions. Complete response (CR) is defined as disappearance of all target lesions. Any pathological lymph nodes (whether target or non-target) must display a reduction in the short axis to $<10 \mathrm{~mm}$. Partial response (PR) is defined as at least a $30 \%$ decrease in the sum of the diameters of the target lesions as compared with the baseline sum diameters. Progressive disease (PD) is defined as at least a 20\% increase in the sum of the diameters of the target lesions compared with the smallest sum in the study (this includes the baseline sum, if that is the smallest). In addition to the relative increase of $20 \%$, the sum must also exhibit an absolute increase of at least $5 \mathrm{~mm}$ (of note, the appearance of one or more new lesions is also considered as progression). Finally, stable disease (SD) is defined as neither sufficient shrinkage to qualify for PR, nor sufficient increase in size to qualify for PD as compared with the smallest sum diameters in the study.

$m G P S$. The GPS has been reported to be a useful inflammatory index for assessing the status of cachexia. This score is composed of C-reactive protein (CRP) levels to reflect the systemic inflammation status, and serum albumin levels to reflect the nutritional status (14). At present, mGPS is widely used to classify patients into three groups, namely 0,1 and 2 . mGPS was calculated as follows: $0, \mathrm{CRP} \leq 1.0 \mathrm{mg} / \mathrm{dl} ; 1, \mathrm{CRP}$ $>1.0 \mathrm{mg} / \mathrm{dl}$; and 2, CRP $>1.0 \mathrm{mg} / \mathrm{dl}$ and albumin $<3.5 \mathrm{mg} / \mathrm{dl}(15)$.

Statistical analysis. All data are presented as the mean \pm standard deviation. The Student's t-test, Wilcoxon's signed-rank test, Kaplan-Meier method, log-rank test, and Pearson's product-moment correlation coefficient were used to evaluate the data and determine statistical significance. $\mathrm{P}<0.05$ was considered to indicate a statistically significant difference. All statistical analyses were performed with SPSS 11.5J software (SPSS Japan, Inc.).

\section{Results}

Study population. The present study included 17 patients with mCRC (12 men and 5 women; mean age, 60.4 \pm 13.4 years; range, 41-81 years), all of whom had an Eastern Cooperative Oncology Group performance status of 0-2. The study population was heavily pretreated: The majority (71\%) had received $\geq 4$ prior regimens and, in addition to fluoropyrimidine, irinotecan and oxaliplatin, all had received bevacizumab (100\%) and either cetuximab or panitumumab (47\%). The RAS gene mutation status was determined for all 17 patients: $9(53 \%)$ had wild-type and 8 (47\%) had mutant RAS (Table II). All 17 patients received TAS-102 plus bevacizumab at the dosages 
Table I. Baseline characteristics (before salvage-line treatment).

\begin{tabular}{lc}
\hline $\begin{array}{l}\text { Previous chemotherapy and } \\
\text { reason for discontinuation }\end{array}$ & $\begin{array}{c}\text { All patients } \\
(\mathrm{n}=17), \text { no. }(\%)\end{array}$ \\
\hline Fluoropyrimidine & $17(100)$ \\
Refractory & 0 \\
Intolerant & \\
Oxaliplatin & $16(94)$ \\
Refractory & $1(6)$ \\
Intolerant & \\
Irinotecan & $15(88)$ \\
Refractory & $2(12)$ \\
Intolerant & \\
Angiogenesis inhibitor & $17(100)$ \\
Refractory & 0 \\
Intolerant & \\
Anti-EGFR antibody & \\
Refractory & $8(47)$ \\
Intolerant & $1(6)$ \\
RAS mutational status & \\
Wild-type & $9(53)$ \\
Mutant & $8(47)$ \\
\hline
\end{tabular}

EGFR, epidermal growth factor receptor.

described in Patients and methods. All patient had undergone primary tumor resection prior to salvage-line treatment.

Adverse events (classified according to the National Cancer Institute Common Terminology Criteria for Adverse Events, version 5.0) were observed in all 17 patients (16). Grade 3/4 toxicity was predominantly hematological, consisting mostly of neutropenia (41\%), leukopenia (11\%) and anemia (5\%). Febrile neutropenia was not observed in any of the patients. Non-hematological toxicity consisted mainly of fatigue, nausea, proteinuria and anorexia, but it was rarely grade $\geq 3$. There were no reported treatment-related deaths (Table III).

The first staging imaging scans ( after 2 cycles of therapy) were available for review in all 17 patients. At the first evaluation, 5 (29\%) patients had PD, 12 (71\%) patients had SD, whereas none had PR to TAS-102 plus bevacizumab (Table IV). The respective median OS and progression-free survival (PFS) times were 14.1 and 6.8 months, respectively, which were somewhat better compared with the respective 11.2 and 5.6 months reported in the C-TASK FORCE study (12) (Figs. 1 and 2).

Furthermore, patients with $\mathrm{mCRC}$ receiving salvage-line therapy who developed chemotherapy-induced neutropenia at 1 month (CIN-1-month) had significantly improved PFS (grade 0-2 vs. 3-4: 2.7 vs. 12.1 months, respectively; $\mathrm{P}=0.021$ ) (Fig. 3). Unfortunately, individuals who developed CIN-1-month exhibited no significant improvement in OS (Fig. 4).

The median PFS was significantly different among the three mGPS groups (mGPS 0 vs. mGPS 2, $\mathrm{P}=0.02$; mGPS 1 vs. $\mathrm{mGPS} 2, \mathrm{P}=0.06$ ). The median PFS in the mGPS 0,1 and 2 groups was 12.1, 4.8 and 2.3 months, respectively. The
Table II. Patient characteristics.

\begin{tabular}{lc}
\hline Characteristics & All patients \\
$(\mathrm{n}=17)$, no. $(\%)$
\end{tabular}

SD, standard deviation; ECOG, Eastern Cooperative Oncology Group.

median OS was also significantly among the three groups (mGPS 0 vs. mGPS 2, $\mathrm{P}=0.01$; mGPS 1 vs. mGPS 2, $\mathrm{P}=0.04$ ). The median OS in the mGPS 0,1 and 2 groups was 14.0 , not reached, and 4.3 months, respectively (Figs. 5 and 6).

\section{Discussion}

Recently, chemotherapy for CRC has markedly progressed. In particular, the treatment for advanced or metastatic CRC has significantly improved due to the development of the FOLFOX and FOLFIRI regimens. Furthermore, the introduction of targeted therapy has further increased the effectiveness of CRC treatment (17-20).

The development of molecular targeted agents has contributed to the prolongation of $\mathrm{mCRC}$ patient survival. One anti-VEGF agent, bevacizumab, and two anti-epidermal growth factor receptor (EGFR) agents, cetuximab and panitumumab, have shown clinical benefits in the first-, second- and salvage-line setting in combination with cytotoxic chemotherapy. Moreover, RAS mutations have been proven to be a negative biomarker for anti-EGFR therapy in recent retrospective analyses $(6,21)$.

As salvage-line chemotherapy, regorafenib was the first small-molecule multikinase inhibitor to offer a survival benefit in $\mathrm{mCRC}$ that has progressed after all standard 
Table III. Frequency of adverse events and laboratory abnormalities.

\begin{tabular}{lcc}
\hline & \multicolumn{2}{c}{ TFTD $+\mathrm{BV}, \mathrm{n}=17(\%)$} \\
\cline { 2 - 3 } & $\begin{array}{c}\text { All grade, } \\
\mathrm{n}(\%)\end{array}$ & $\begin{array}{c}\text { Grade } 3 / 4, \\
\mathrm{n}(\%)\end{array}$ \\
Adverse events & & \\
\hline Hematological & $11(64)$ & $7(41)$ \\
Neutropenia & $11(64)$ & $1(11)$ \\
Leukopenia & $8(47)$ & $1(5)$ \\
Anemia & & \\
Non-hematological & $7(41)$ & $1(5)$ \\
Increased blood bilirubin & $7(41)$ & $1(5)$ \\
Increased ALT & $6(35)$ & $1(5)$ \\
Increased AST & $6(35)$ & $1(5)$ \\
Increased ALP & $0(0)$ & $0(0)$ \\
Febrile neutropenia & $5(29)$ & $1(5)$ \\
Hypertension & $7(41)$ & $0(0)$ \\
Anorexia & $2(11)$ & $0(0)$ \\
Oral mucositis & $9(52)$ & $0(0)$ \\
Proteinuria & $8(47)$ & $0(0)$ \\
Nausea & $8(47)$ & $0(0)$ \\
Diarrhea & $2(11)$ & $1(5)$ \\
Vomiting & $10(58)$ & $0(0)$ \\
Fatigue & $5(11)$ & $1(5)$ \\
Fever & $5(29)$ & \\
Skin rash & & \\
Epistaxis & & \\
\hline
\end{tabular}

TFTD, trifluridine and tipiracil hydrochloride; BV, bevacizumab; ALT, alanine aminotransferase; AST, aspartate aminotransferase; ALP, alkaline phosphatase.

Table IV. Best response to treatment.

\begin{tabular}{lc}
\hline Response to treatment & TFTD + BV, $\mathrm{n}=17(\%)$ \\
\hline Complete response (CR) & $0(0)$ \\
Partial response (PR) & $0(0)$ \\
Stable disease (SD) & $12(71)$ \\
Progressive disease (PD) & $5(29)$ \\
Not evaluable (NE) & $0(0)$ \\
Overall response (CR + PR) & $0(0)$ \\
Disease control (CR + PR + SD) & $12(71)$ \\
\hline
\end{tabular}

TFTD, trifluridine and tipiracil hydrochloride; BV, bevacizumab.

therapies have been exhausted. In the CORRECT study, no patients achieved a CR; however, 5 patients receiving regorafenib and 1 patient assigned to placebo exhibited a PR, with objective response rates of 1.0 and $0.4 \%$, respectively $(\mathrm{P}=0.19)$. As $C R$ and $P R$ were obtained in only a few patients, regorafenib is unlikely to fully achieve a complete or partial
Progression-free survival 29.6 weeks ( 6.8 months)

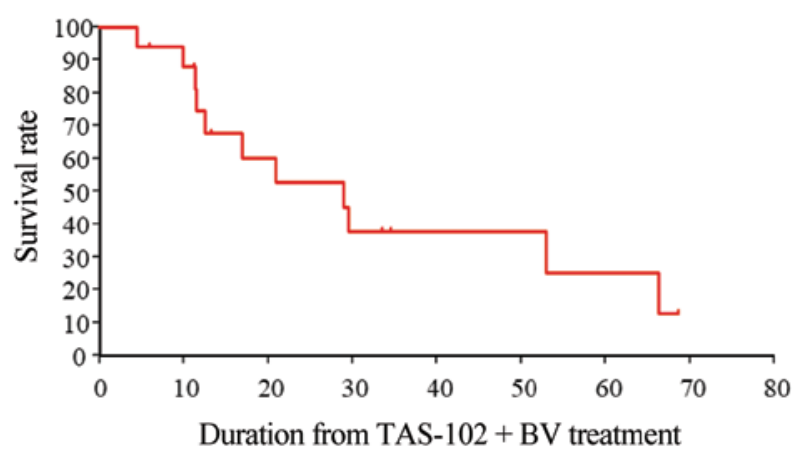

Figure 1. Kaplan-Meier curves of investigator-assessed progression-free survival $(\mathrm{n}=17)$

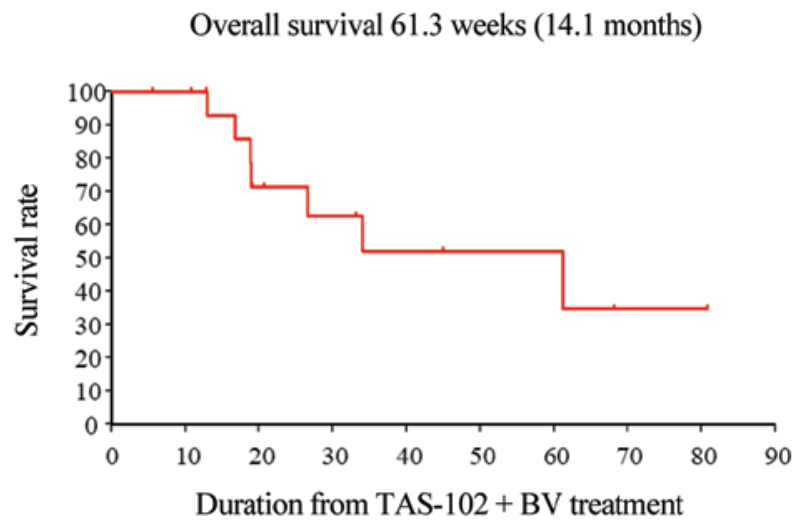

Figure 2. Kaplan-Meier curves of investigator-assessed overall survival $(n=17)$.

Progression-free survival Grade 0-2 MST 12.6 weeks (2.7 months) Grade 3-4 MST 53.0 weeks (12.1 months)

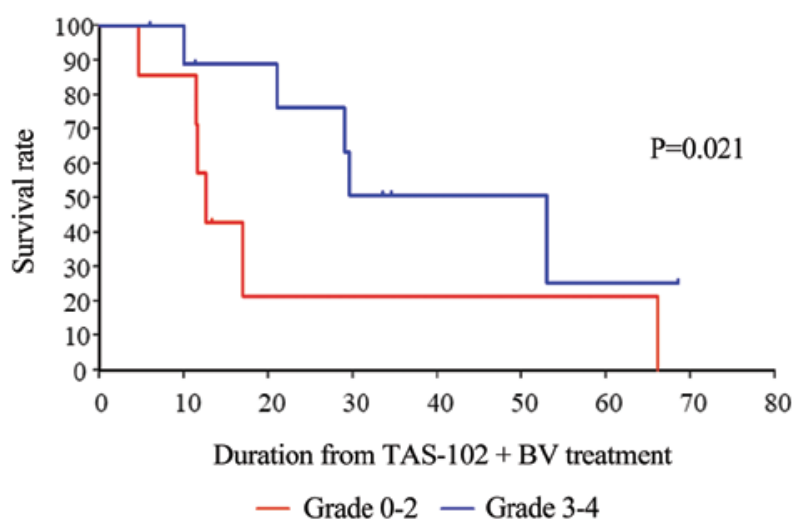

Figure 3. Kaplan-Meier curves of PFS in patients with metastatic colorectal cancer who developed chemotherapy-induced neutropenia (CIN) in the salvage-line setting revealed that patients with CIN-1-month had significantly improved survival (grade $0-2$ vs. 3-4: 2.7 vs. 12.1 months, respectively; $\mathrm{P}=0.021)$. PFS, progression-free survival.

disease response. However, disease control was achieved in $41 \%$ of the patients assigned regorafenib and in $15 \%$ of the patients assigned placebo $(\mathrm{P}<0.0001)$. The median duration of 


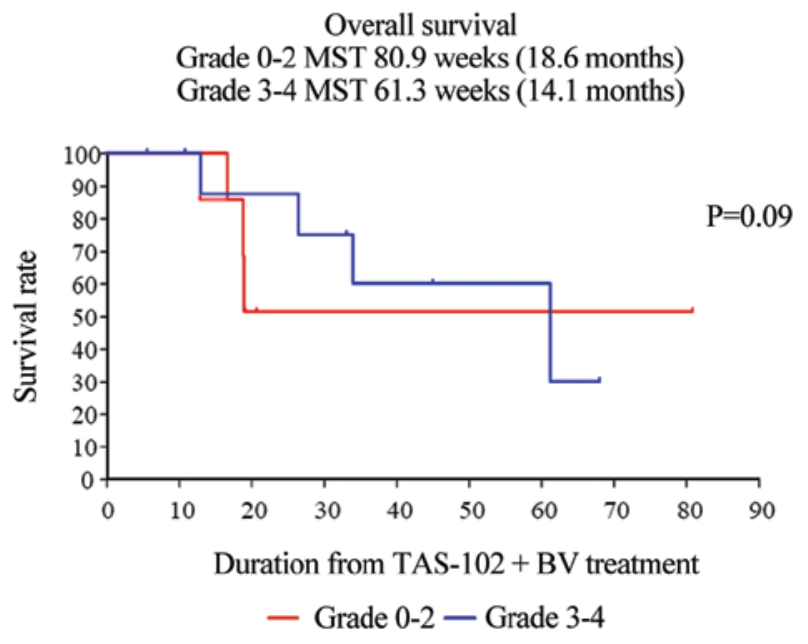

Figure 4. Kaplan-Meier curves of OS in patients with metastatic colorectal cancer who developed chemotherapy-induced neutropenia (CIN) revealed that patients with CIN-1-month did not have a significant improvement in survival. OS, overall survival.

Progression-free survival GPS 0 MST 53.0 weeks (12.1 months) GPS 1 MST 21.0 weeks ( 4.8 months) GPS 2 MST 10.0 weeks (2.3 months)

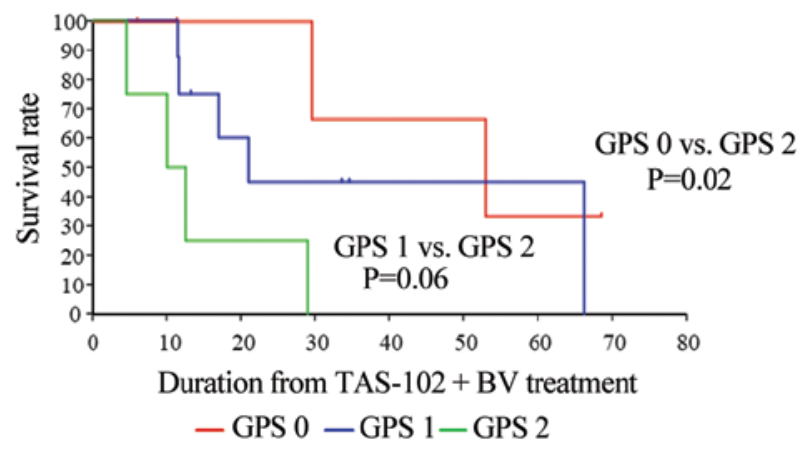

Figure 5. Kaplan-Meier curves demonstrated that the median PFS was significantly different among mGPS groups (mGPS 0 vs. mGPS $2, \mathrm{P}=0.02$; mGPS 1 vs. mGPS 2, P=0.06). The median PFS in the mGPS 0,1 and 2 groups was 12.1, 4.8 and 2.3 months, respectively. PFS, progression-free survival; mGPS, modified Glasgow Prognostic Score.

SD was 2.0 months in the regorafenib group and 1.7 months in the placebo group (22).

In salvage-line treatment of mCRC, TAS-102 was reported to significantly improve OS compared with placebo in the RECOURSE trial. In the tumor-response population (502 patients in the TAS-102 group and 258 in the placebo group), 8 patients in the TAS-102 group had a $\mathrm{PR}$, and 1 patient in the placebo group had a CR, resulting in objective response rates of $1.6 \%$ with TAS-102 and $0.4 \%$ with placebo $(\mathrm{P}=0.29)$. Disease control $(\mathrm{CR}, \mathrm{PR}$ or $\mathrm{SD}$, assessed at least 6 weeks after randomization) was achieved in 221 patients $(44 \%)$ in the TAS-102 group and 42 patients $(16 \%)$ in the placebo group $(\mathrm{P}<0.001)$. Patients in the TAS-102 group received the study drug for a mean \pm standard deviation of $12.7 \pm 12.0$ weeks (median, 6.7; range, 0.1-78.0 weeks), and patients in the placebo group received

\section{Overall survival \\ GPS 0 MST 61.2 weeks (14.0 months) \\ GPS 1 MST not reached \\ GPS2 MST 19.0 weeks (4.3 months)}

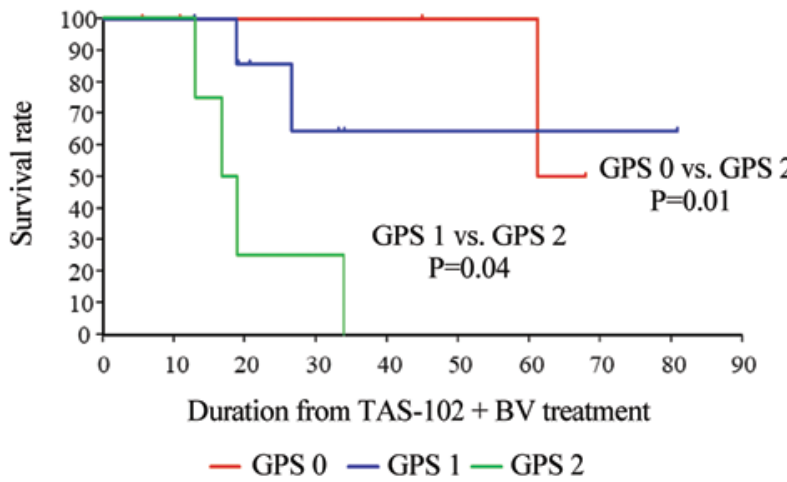

Figure 6. Kaplan-Meier curves demonstrated that the median OS was significantly different among mGPS groups (mGPS 0 vs. mGPS 2, P=0.01; mGPS 1 vs. mGPS 2, $\mathrm{P}=0.04$ ). The median OS in the mGPS 0,1 and 2 groups was 14.0 , not reached, and 2 months, respectively. OS, overall survival; mGPS, modified Glasgow Prognostic Score.

the study drug for a mean of $6.8 \pm 6.1$ weeks (median, 5.7; range, 0.1-63.7 weeks). The assessment of tumor status with regard to KRAS demonstrated that $49 \%$ of the patients had wild-type tumors and $51 \%$ had mutant tumors. A benefit from treatment with TAS-102 was observed in both patient subgroups (20). In the CORRECT trial, treatment-related adverse events occurred in $93 \%$ patients receiving regorafenib and in $61 \%$ of those receiving placebo. The most common adverse events of grade $\geq 3$ associated with regorafenib were hand-foot skin reaction (17\%), fatigue $(10 \%)$, diarrhea $(n=36,7 \%)$, hypertension $(n=36,7 \%)$, and rash or desquamation $(n=29,6 \%)(22)$.

Although regorafenib and TAS-102 are effective as salvage-line therapies, OS and PFS remain unsatisfactory with these treatments. Recently, the phase I/II C-TASK FORCE trial was conducted to investigate the efficacy and safety of TAS-102 plus bevacizumab in the salvage-line setting. The median OS was 11.2 months and the PFS was 5.6 months. In addition, although the relative risk was only $4.0 \%$, the disease control rate was $72 \%$, with tolerable toxicity. However, the sample size was quite small $(n=25)$. The assessment of tumor status with regard to KRAS revealed that $49 \%$ of the patients had wild-type and $51 \%$ had mutant tumors. A benefit from treatment with TAS-102 was also observed in both patient subgroups (12).

At present, there are almost no reports on the antitumor effect of combined TAS-102 and bevacizumab. Tsukihara et al evaluated the mechanism underlying the enhanced antitumor effect of combined TAS-102 and bevacizumab (23). They measured trifluridine (FTD) and its phosphorylated forms in tumors, as these are the active components and metabolites of TAS-102. Phosphorylated FTD levels were increased by combining TAS-102 and bevacizumab in both SW48 and HCT116 tumors (23). In addition, Jain et al reported that tumor blood vessels are generally poorly organized and hyperpermeable, with an impaired gradient between the vascular and interstitial pressure and, consequently, offer a diminished blood supply (24). They further reported that these vessels may 
also limit the accumulation of FTD in tumors. Bevacizumab inhibits angiogenesis by antagonizing VEGF, and may therefore normalize tumor vasculature, thus improving tumor blood supply and increasing FTD accumulation and its subsequent phosphorylation in the tumor.

The combination regimen of a fluoropyrimidine with bevacizumab in $\mathrm{mCRC}$ in the first-line setting was established on the basis of high clinical effectiveness and no overlapping toxicity between agents. It is considered that sustained VEGF inhibition achieves and maintains tumor regression. The addition of bevacizumab, aflibercept, or ramucirumab to chemotherapy has shown a survival benefit in mCRC in the second-line setting $(25,26)$. However, little is known on the efficacy and safety of continued administration of angiogenesis inhibitors with cytotoxic chemotherapy beyond progression after second-line therapy. Bevacizumab combined with a chemotherapy regimen has a fully manageable toxicity profile. From these viewpoints, combining TAS-102 with bevacizumab may prove to be beneficial.

Kasi et al reported that CIN-1-month after starting TAS-102 appears to be a prognostic and/or predictive biomarker of both PFS and OS in patients with mCRC undergoing salvage-line therapy (26). In the present study, patients who developed CIN-1-month had significantly improved PFS (grade 0-2 vs. 3-4: 2.7 vs. 12.1 months, respectively; $\mathrm{P}=0.021$ ), although $\mathrm{OS}$ was not significantly improved (data not shown).

Parallel to the increase in the serum CRP level, hypoalbuminemia has been observed in various types of tumors and verified as a negative prognostic factor (27-29). The association between hypoalbuminemia and reduced survival in patients with cervical cancer is affected by several factors. Hypoalbuminemia reflects a progressive nutritional decline in cancer patients, and is associated with cancer-related cachexia (30).

The GPS was shown to be a powerful prognostic factor. This cumulative prognostic score is based on pre-treatment values of CRP and albumin, is objective, and has shown superior prognostic value in various cancers. A correlation between preoperative GPS and CRC survival has been reported (31). In the same manner, GPS was shown to be a powerful prognostic factor in cancer patients, irrespective of tumor site (32). Whether the prognostic value of the GPS is modified by the addition of non-cytotoxic agents with high activity and tolerable toxicity, such as salvage-line TAS-102 plus bevacizumab, remains unknown. In our experience, mGPS allowed prediction of PFS and OS in patients with $\mathrm{mCRC}$ receiving salvage-line therapy, such as TAS-102 plus bevacizumab.

In the present study, the median PFS was significantly different for each factor of CIN-1 and each mGPS group, whereas the median OS was not significantly different. However, in a study of this sample size, it may be concluded that this result is important as real world data in future prospective studies. mGPS as an inflammatory index is also a useful predictor marker in the salvage-line setting.

The results of the present study have shown that in salvage-line therapy for patients with $\mathrm{mCRC}$, bevacizumab enhances the antitumor effect of TAS-102, with superior median OS and PFS times compared with those reported in the C-TASK FORCE study.
In conclusion, a clinical study of combined TAS-102 and bevacizumab therapy is currently in phase II and phase III, and we expect its outcome to be highly informative. Although the sample size of the present study was small, the findings confirm the prognostic accuracy of the mGPS score in salvage-line therapy for patients with $\mathrm{mCRC}$.

\section{Acknowledgements}

Not applicable.

\section{Funding}

No funding was received.

\section{Availability of materials and data}

All the datasets generated and analyzed in the present study are available from the corresponding author on reasonable request.

\section{Authors' contributions}

NM, HF and TT conceived and designed the study. NM, TT, HF, TS, MF, YI, YT, RM, ToT, SM, HI, YT, KYa, MF and KYo acquired the data. NM analyzed and interpreted the data and drafted the manuscript. NM, TT and KYo performed critical revision of the manuscript. KYo supervised the study. All authors have read and approved the final version of the manuscript.

\section{Ethics approval and consent to participate}

All procedures performed in studies involving human participants were in accordance with the ethical standards of the institutional and/or national research committee and with the 1975 Helsinki declaration and its later amendments or comparable ethical standards. Informed consent was obtained from all individual participants included in the study.

\section{Patient consent for publication}

Not applicable.

\section{Competing interests}

K. Yoshida has received honoraria for lectures from Chugai Pharmaceutical Co., Ltd., Taiho Pharmaceutical Co., Ltd., Takeda Pharmaceutical Co., Ltd., Eli Lilly and Company, Daiichi Sankyo Co., Ltd., Ono Pharmaceutical Co., Ltd., Merck Serono Co., Ltd., Novartis Pharma K.K., and Sanofi K.K.; and research funding from Ajinomoto Pharmaceutical Co., Ltd., Takeda Pharmaceutical Co., Ltd., Chugai Pharmaceutical Co., Ltd., Daiichi Sankyo Co., Ltd., Taiho Pharmaceutical Co., Ono Pharmaceutical Co., and Yakult Honsha Co., Ltd. outside the submitted work.

T. Takahashi has received honoraria for lectures from Takeda Pharmaceutical Co., Ltd. All remaining authors declare that they have no conflicts of interest. 


\section{References}

1. Center MM, Jemal A and Ward E: International trends in colorectal cancer incidence rates. Cancer Epidemiol Biomarkers Prev 18: 1688-1694. 2009

2. Cheng AL, Li J, Vaid AK, Ma BB, The C, Ahn JB, Bello M, Charoentum C, Chen LT, de Lima Lopes G Jr, et al: Adaptation of international guidelines for metastatic colorectal cancer: An Asian consensus. Clin Colorectal Cancer 13: 145-155, 2014

3. Bray F, Ferlay J, Soerjomataram I, Siegel RL, Torre LA and Jemal A: Global cancer statistics 2018: GLOBOCAN estimates of incidence and mortality worldwide for 36 cancersin 185 countries. CA Cancer J Clin 68: 394-424, 2018.

4. Lemmens V, van Steenbergen L, Janssen-Heijnen M, Martijn H, Rutten $\mathrm{H}$ and Coebergh JW: Trends in colorectal cancer in the south of the Netherlands 1975-2007: rectal cancer survival levels with colon cancer survival. Acta Oncol 49: 784-796, 2010.

5. Van Cutsem E, Borràs JM, Castells A, Ciardiello F, Ducreux M, Haq A, Schmoll HJ and Tabernero J: Improving outcomes in colorectal cancer: where do we go from here? Eur J Cancer 49: 2476-2485, 2013

6. Van Cutsem E, Köhne CH, Hitre E, Zaluski J, Chang Chien CR, Makhson A, D'Haens G, Pintér T, Lim R, Bodoky G, et al: Cetuximab and chemotherapy as initial treatment for metastatic colorectal cancer. N Engl J Med 360: 1408-1417, 2009.

7. Yamada Y, Denda T, Gamoh M, Iwanaga I, Yuki S, Shimodaira H, Nakamura M, Yamaguchi T, Ohori H, Kobayashi K, et al: S-1 and irinotecan plus bevacizumab vs. mFOLFOX6 or CapeOX plus bevacizumab as first-line treatment in patients with metastatic colorectal cancer (TRICOLORE): A randomized, open-label, phase III, noninferiority trial. Ann Oncol 29: 624-631, 2018.

8. Van Cutsem E, Cervantes A, Adam R, Sobrero A, Van Krieken JH, Aderka D, Aranda Aguilar E, Bardelli A, Benson A, Bodoky G, et al: ESMO consensus guidelines for the management of patients with metastatic colorectal cancer. Ann Oncol 27: 1386-1422, 2016

9. Mayer RJ, Van Cutsem E, Falcone A, Yoshino T, Garcia-Carbonero R, Mizunuma N, Yamazaki K, Shimada Y, Tabernero J, Komatsu Y, et al: RECOURSE Study Group. Randomized trial of TAS-102 for refractory metastatic colorectal cancer. N Engl J 372: 1909-1919, 2015.

10. Melnyk O,Zimmerman M, Kim KJ and Shuman M: Neutralizing anti-vascular endothelial growth factor antibody inhibits further growth of established prostate cancer and metastases in a pre-clinical model. J Urol 161: 960-963, 1999.

11. Klement G,Baruchel S, Rak J,Man S,Clark K, Hicklin DJ, Bohlen P and Kerbel RS: Continuous low-dose therapy with vinblastine and VEGF receptor-2 antibody induces sustained tumor regression without overt toxicity. J Clin Invest 105: R15-R24, 2000.

12. Kuboki Y, Nishina T, Shinozaki E, Yamazaki K, Shitara K, Okamoto W, Kajiwara T, Matsumoto T, Tsushima T, Mochizuki N, et al: TAS-102 plus bevacizumab for patients with metastatic colorectal cancer refractory to standard therapies (C-TASK FORCE): An investigator-initiated, open-label, single-arm, multicentre, phase 1/2 study. Lancet Oncol 18: 1172-1181, 2017.

13. Yoshino T, Oki E, Nozawa H, Eguchi-Nakajima T, Taniguchi H, Morita S, Takenaka N, Ozawa D and Shirao K: Rationale and design of the TRUSTY study: A randomised, multicentre, open-label phase II/III study of trifluridine/tipiracil plus bevacizumab versus irinotecan, fluoropyrimidine plus bevacizumab as second-line treatment in patients with metastatic colorectal cancer progressive during or following first-line oxaliplatin-based chemotherapy. ESMO Open 3: e000411, 2018.

14. Douglas E and McMillan DC: Towards a simple objective framework for the investigation and treatment of cancer cachexia: The glasgow prognostic score. Cancer Treat Rev 40: 685-691, 2014.

15. Takeno S, Hashimoto T, Shibata R, Maki K, Shiwaku H, Yamana I, Yamashita R and Yamashita Y: The high-sensitivity modified Glasgow prognostic score is superior to the modified Glasgow prognostic score as a prognostic predictor in patients with resectable gastric cancer. Oncology 87: 205-214, 2014

16. Common Terminology Criteria for Adverse Events (CTCAE) Version5.0 Published Nov 27, 2017.

17. Douillard JY, Cunningham D, Roth AD, Navarro M, James RD, Karasek P, Jandik P, Iveson T, Carmichael J, Alakl M, et al: Irinotecan combined with fluorouracil compared with fluorouracil alone as first-line treatment for metastatic colorectal cancer: A multicentre randomised trial. Lancet 355: 1041-1047, 2000 .
18. Tournigand C, André T, Achille E, Lledo G, Flesh M, Mery-Mignard D, Quinaux E, Couteau C, Buyse M, Ganem G, et al: FOLFIRI followed by FOLFOX6 or the reverse sequence in advanced colorectal cancer: A randomized GERCOR study. J Clin Oncol 22: 229-237, 2004

19. Falcone A, Ricci S, Brunetti I, Pfanner E, Allegrini G, Barbara C, Crinò L, Benedetti G, Evangelista W, Fanchini L, et al: Gruppo Oncologico Nord Ovest.Phase III trial of infusional fluorouracil, leucovorin, oxaliplatin, and irinotecan (FOLFOXIRI) compared with infusional fluorouracil, leucovorin, and irinotecan (FOLFIRI) as first-line treatment for metastatic colorectal cancer: The Gruppo Oncologico Nord Ovest. J Clin Oncol 25: 1670-1676, 2007.

20. Grothey A, Sargent D, Goldberg RM and Schmoll HJ: Survival of patients with advanced colorectal cancer improves with the availability of fluorouracil-leucovorin, irinotecan, and oxaliplatin in the course of treatment. J Clin Oncol 22: 1209-1214, 2004

21. Hurwitz H, Fehrenbacher L, Novotny W, Cartwright T, Hainsworth J, Heim W, Berlin J, Baron A, Griffing S, Holmgren E, et al: Bevacizumab plus irinotecan, fluorouracil, and leucovorin for metastatic colorectal cancer. N Engl J Med 350: 2335-2342, 2004

22. Grothey A, Van Cutsem E, Sobrero A, Siena S, Falcone A, Ychou M, Humblet Y, Bouché O, Mineur L, Barone C, et al: Regorafenib monotherapy for previously treated metastatic colorectal cancer (CORRECT): An international, multicenter, randomised, placebo-controlled phase 3 trial. Lancet 381: 303-312, 2013.

23. Tsukihara H, Nakagawa F, Sakamoto K, Ishida K, Tanaka N, Okabe H, Uchida J, Matsuo K and Takechi T: Efficacy of combination chemotherapy using a novel oral chemotherapeutic agent, TAS-102, together with bevacizumab, cetuximab, or panitumumab on human colorectal cancer xenografts. Oncol Rep 33: 2135-2142, 2015

24. Jain RK: Normalizing tumor vasculature with anti-angiogenic therapy: A new paradigm for combination therapy. Nat Med 7: 987-989, 2001

25. Van Cutsem E, Tabernero J, Lakomy R, Prenen H, Prausová J, Macarulla T, Ruff P, van Hazel GA, Moiseyenko V, Ferry D, et al: Addition of aflibercept to fluorouracil, leucovorin, and irinotecan improves survival in a phase III randomized trial in patients with metastatic colorectal cancer previously treated with an oxaliplatin-based regimen. J Clin Oncol 30: 3499-3506, 2012

26. Tabernero J, Yoshino T, Cohn AL, Obermannova R, Bodoky G, Garcia-Carbonero R, Ciuleanu TE, Portnoy DC, Van Cutsem E, Grothey A, et al: Ramucirumab versus placebo in combination with second-line FOLFIRI in patients with metastatic colorectal carcinoma that progressed during or after first-line therapy with bevacizumab, oxaliplatin, and a fluoropyrimidine (RAISE): A randomised, double-blind, multicentre, phase 3 study. Lancet Oncol 16: 499-508, 2015.

26. Kasi PM, Kotani D, Cecchini M, Shitara K, Ohtsu A, Ramanathan RK, Hochster HS, Grothey A and Yoshino T: Chemotherapy induced neutropenia at 1-month mark is a predictor of overall survival in patients receiving TAS-102 for refractory metastatic colorectal cancer: A cohort study. BMC Cancer 16: 467, 2016.

27. McMillan DC: An inflammation-based prognostic score and its role in the nutrition-based management of patients with cancer. Proc Nutr Soc 67: 257-262, 2008.

28. Deans DA, Tan BH, Wigmore SJ, Ross JA, de Beaux AC, Paterson-Brown S and Fearon KC: The influence of systemic inflammation, dietary intake and stage of disease on rate of weight loss in patients with gastro-oesophageal cancer. $\mathrm{Br} J$ Cancer 100: 63-69, 2009.

29. Morley JE, Thomas DR and Wilson MM: Cachexia: Pathophysiology and clinical relevance. Am J Clin Nutr 83: 735-743, 2006.

30. Ishizuka $M$, Nagata $H$, Takagi $K$, Horie $T$ and Kubota $K$ : Inflammation-based prognostic score is a novel predictor of postoperative outcome in patients with colorectal cancer. Ann Surg 246: 1047-1051, 2007.

31. Proctor MJ, Morrison DS, Talwar D, Balmer SM, O'Reilly DS, Foulis AK, Horgan PG and McMillan DC: An inflammationbased prognostic score (mGPS) predicts cancer survival independent of tumour site: A glasgow inflammation outcome study. Br J Cancer 104: 726-732, 2011.

32. Prager GW, Braemswig KH, Martel A, Unseld M, Heinze G, Brodowicz T, Scheithauer W, Kornek G and Zielinski CC: Baseline carcinoembryonic antigen (CEA) serum levels predict bevacizumab-based treatment response in metastatic colorectal cancer. Cancer Sci 105: 996-1001, 2014 